\title{
A small GTPase-like protein fragment of Mycoplasma promotes tumor cell migration and proliferation in vitro via interaction with Rac1 and Stat3
}

\author{
XIAOPENG HU ${ }^{1 *}$, JIE YU $^{2 *}$, XIANG ZHOU $^{3}$, ZHAOMING LI $^{4}$, YUN XIA $^{1}$ \\ ZHIYONG LUO ${ }^{1}$ and YAQUN WU ${ }^{1}$
}

\begin{abstract}
${ }^{1}$ Department of General Surgery, Tongji Hospital, Tongji Medical College, Huazhong University of Science and Technology, Wuhan, Hubei 430030; ${ }^{2}$ First People's Hospital of Jiujiang City, Jiujiang, Jiangxi 330300; ${ }^{3}$ The Fifth Hospital of Huangshi City, Huangshi, Hubei 435004; ${ }^{4}$ Department of Oncology, The First Affiliated Hospital,

Zhengzhou University, Zhengzhou, Henan 450052, P.R. China
\end{abstract}

Received April 1, 2013; Accepted October 21, 2013

DOI: $10.3892 / \mathrm{mmr} .2013 .1766$

\begin{abstract}
The Mycoplasma genus comprises a group of microbes that cause persistent infection in humans and its role in promoting tumor development has long been a concern. Although mixtures of components isolated from Mycoplasma have been shown to activate host Rho family small GTPases and Stat3, no individual factor with this activity has been reported. In the current study, a conserved small GTPase-like protein fragment (SGLP) from Mycoplasma pulmonis chromosome partition protein, Smc, was identified as a virulence factor. SGLP was observed to interact with Rac1 and Stat3. The wild-type (wt) SGLP, which contains a WxxxE motif, induced activation of Rac1 and phosphorylation of Stat 3 at the tyrosine-705 residue, while the SGLP mutant containing a mutation from WxxxE to AxxxA did not exert the same effects. Moreover, SGLP-induced Stat3 phosphorylation was observed to be dependent upon Rac1 activity. Furthermore, wt SGLP was observed to promote cell migration and increase bromodeoxyuridine incorporation in HeLa cells and the SGLP mutant did not elicit these effects in HeLa cells. In conclusion, the current observations suggest that SGLP is an important virulence factor of Mycoplasma, which contributes to tumor cell migration and proliferation in vitro via interaction with Rac1 and Stat3.
\end{abstract}

Correspondence to: Dr Xiaopeng $\mathrm{Hu}$, Department of General Surgery, Tongji Hospital, Tongji Medical College, Huazhong University of Science and Technology, 1095 Jiefang Avenue, Wuhan, Hubei 430030, P.R. China

E-mail: xiaopeng_hu@hust.edu.cn

${ }^{*}$ Contributed equally

Key words: Mycoplasma, tumor cell, Rac1, Stat3

\section{Introduction}

Persistent inflammatory microenvironment induced by bacteria, including Mycoplasma, is conducive to tumor development in host tissues (1). Mycoplasma promotes multistage malignant transformation of host cells following a long-term infection $(2,3)$. Being different from other bacteria, Mycoplasma lacks a rigid cell wall, which facilitates fusion with the host cell membrane (4). Following fusion, Mycoplasma may lead to various alterations in the affected cells, which involves the Mycoplasma membrane components inserting into the host cell membrane. Among those mycoplasmal components, the lipid-associated membrane proteins have been reported to activate the Jak/Stat3 pathway through mediating host small GTPases of the Rho family, including Rac1 $(5,6)$.

Racl is a significant member of Rho-family small GTPases, which are pleiotropic in controlling actin cytoskeleton reorganization (7) and Stat3 is an important transcription factor that may be activated by the GTP-bound form of active Rac1 (8-10). In terms of subcellular localization, active Rac1 contributes to Stat3 nuclear translocation by forming a complex with a Rac1/Cdc42 GTPase-activating protein $(11,12)$, whereas cytoplasmic Stat3 regulates Rac1 activity to modulate directional cell migration (13). The functional and physical interactions between Rac1 and Stat 3 potentiate cell proliferation and migration in certain cell types $(8,13-15)$.

There are a number of bacterial virulence factors that possess a WxxxE motif, which may subvert cellular functions via interacting with host small GTPases of the Rho family. These identified factors include: The Shigella effectors, IpgB1 and IpgB2 (16), the E. coli effector, Map (17), the Salmonella effector, SifA (18) and Pantoea stewartii subsp. stewartii and Pseudomonas syringae effectors, WtsE and AvrE1, respectively (19).

Through computational analysis of Mycoplasma genomes, the chromosome partition protein Smc (Smc) conserved among Mycoplasma species was observed to possess an invariant WxxxE motif at its $\mathrm{N}$ terminus, which is associated with Rho family small GTPases, as well as a probable coiled 
coil domain downstream of the WxxxE motif, which may interact with Stat 3 as predicted by an online program designed for predicting the parallel coiled coil interaction (20). Notably, Stat 3 is a binding partner of Rac1 (8); therefore, the present study aimed to determine whether the N-terminal domain of Smc interacts with Rac1 and/or Stat3.

The N-terminal sequence (amino acids 9-248) of Smc (UniProt: Q98PK8) was cloned from Mycoplasma pulmonis, a common Mycoplasma contaminant in cell cultures. The cloned 240-amino acid $\mathrm{N}$-terminus of Smc possesses a characteristic GTP-binding motif of GxxxxGKS/T that is observed in all small GTPases. With regard to such a similarity with small GTPases, this small GTPase-like protein fragment of 240 amino acids from Smc is referred to as SGLP.

In the current study, the interaction of SGLP with Rac1 and Stat 3 was investigated and the mechanism by which SGLP may be involved in affecting tumor cell migration and proliferation through interaction with Rac1 and Stat3 was demonstrated.

\section{Materials and methods}

Antibodies. The primary antibodies used were: p-Stat3 (9145) from Cell Signaling Technology, Inc. (Beverly, MA, USA); glutathione S-transferase (GST; sc-33614) and Rac1 (sc-217) from Santa Cruz Biotechnology, Inc. (Santa Cruz, CA, USA); FLAG (T510-2) and green fluorescent protein (GFP; T508-2) from Signalway Antibody (Baltimore, MD, USA); Myc-Tag (AM1007a) from Abgent (San Diego, CA, USA); anti-bromodeoxyuridine (BrdU; 560810) from BD Biosciences (San Jose, CA, USA) and Stat3 (51076-2-AP) from Proteintech Group (Chicago, IL, USA). Other antibodies used, including FITC-conjugated goat anti-mouse, Alexa Fluor 488-conjugated goat anti-rabbit, Alexa Fluor 594-conjugated goat anti-mouse antibodies and HRP-conjugated goat antibodies against rabbit and mouse, were purchased from Jiayuan Biotech Co. Ltd. (Wuhan, China).

Overlapping polymerase chain reaction $(P C R)$ and plasmid construction. The point mutation for tryptophan and the mutation of WVLGE to AVLGA were generated by overlapping PCR using primers as follows: i) point mutation for tryptophan of SGLP, N-terminus 5'-ATGGATCCAAATCATTTGCAGAGCCAATTC-3' and 5'-GTTCACCCAAGACCCATTTAATGGCATC-3' and C-terminus 5'-GCCATTAAATGGGTCTTGGGTGAA CAAT-3' and 5'-CACCGAATTCCTCAATTTCAAATT CTTTTAGTTTG-3'andii) pointmutationforWxxxEto AxxxA, N-terminus 5'-GATATCAAATCATTTGCAGAGCCAAT-3' and 5'-TGTGCACCCAAGACCGCTTTAATG-3' and C-terminus 5'-AAAGCGGTCTTGGGTGCACAATC-3' and 5'-GCGAATTCCTACTCAATTTCAAATTC-3'. SGLP sequences were cloned into pGEX4T1, pEGFP-C1 and pCDF1-MCS2-EF1-Puro vectors (System Biosciences, Mountain View, CA, USA). In addition, the FLAG-tag DYKDDDDK and Kozak sequences were inserted into the pCDF1-MCS2-EF1-Puro vector. DN-Rac1 (T17N) and CA-Rac1 (Q61L) pRK5 plasmids were purchased from Addgene Inc. (Cambridge, MA, USA).
Cell culture, transfection and stable cell line establishment. HEK 293T and HeLa cells were cultured in Dulbecco's modified Eagle's medium (DMEM; Hyclone, Logan, UT, USA) and supplemented with $10 \%$ fetal bovine serum (FBS; Hyclone) at $37^{\circ} \mathrm{C}$ in a humidified $5 \% \mathrm{CO}_{2}$ atmosphere. Lentiviral packaging was conducted in HEK 293 T cells using pPACK $^{\mathrm{TM}}$ Packaging mix (System Biosciences) according to the manufacturer's instructions. The lentiviral titers were determined by an UltraRapid Titering kit (System Biosciences). HeLa cells were transduced with SGLP lentiviral particles with $6 \mu \mathrm{g} / \mathrm{ml}$ polybrene (Santa Cruz Biotechnology, Inc.) for $12 \mathrm{~h}$. Transduced cells were selected with $2 \mu \mathrm{g} / \mathrm{ml}$ puromycin (Sigma-Aldrich, St. Louis, MO, USA) for two weeks and the stable cell lines were maintained in growth medium with $1 \mu \mathrm{g} / \mathrm{ml}$ puromycin. Lipofectamine ${ }^{\mathrm{TM}} 2000$ (Invitrogen Life Technologies, Carlsbad, CA, USA) was used for transfection of plasmids and small interfering RNAs according to the manufacturer's instructions.

GST pull-down assay, immunoprecipitation and western blot analysis. GST-tagged protein expression was induced by adding $0.1 \mathrm{mM}$ isopropyl- $\beta$-d-thiogalactopyranoside to BL21 E. coli cultures at $30^{\circ} \mathrm{C}$ for $6 \mathrm{~h}$. GST-tagged proteins were purified by affinity chromatography with glutathione Sepharose ${ }^{\text {TM }}$ 4B beads (Amersham Biosciences, Uppsala, Sweden) according to the manufacturer's instructions. HeLa cells $\left(\sim 1 \times 10^{7}\right)$ were treated with $100 \mathrm{ng} / \mathrm{ml} \mathrm{IL}-6$ for $24 \mathrm{~h}$ prior to harvesting. Equal quantities of precleared lysates $(\sim 2 \mathrm{mg})$ were incubated with sepharose beads and $10 \mu \mathrm{g}$ GST or GST-SGLP for $2 \mathrm{~h}$ at $4^{\circ} \mathrm{C}$. The beads were washed three times with washing buffer (pH 7.2, $10 \mathrm{mM} \mathrm{NaPO}_{4}, 10 \mathrm{mM} \mathrm{NaN}_{3}, 150 \mathrm{mM} \mathrm{NaCl}$ and $0.1 \%$ Tween-20). The bait and pray proteins were then eluted from beads with elution buffer ( $\mathrm{pH} 8.0,50 \mathrm{mM}$ Tris- $\mathrm{Cl}$ and $30 \mathrm{mM}$ GSH) and subjected to western blot analysis. The active Rac1 pull-down assay was conducted according to a modified method from Teng et al (13). The GST-Pak1-PBD, which contains the peptide ISLPSDFEHTIHVGF (CRIB domain), was purchased from Millipore (Billerica, MA, USA) and active Rac1, as well as total Rac1, were probed separately. For immunoprecipitation, the GFP- or GFP-SGLP-transfected HeLa cell lysates $(1 \mathrm{mg}$ ) were precleared with $25 \mu \mathrm{l}$ protein $\mathrm{G}$ sepharose beads (Amersham Biosciences) and incubated with $2 \mu \mathrm{g}$ primary antibodies at $4^{\circ} \mathrm{C}$ for $2 \mathrm{~h}$, followed by incubation with $25 \mu \mathrm{l}$ protein $\mathrm{G}$ sepharose beads at $4^{\circ} \mathrm{C}$ for $2 \mathrm{~h}$. Western blot analyses were performed as previously described (21).

Confocal microscopy, fluorescence resonance energy transfer (FRET) and photobleaching analyses. Immunofluorescence images were captured using an Olympus FluoView ${ }^{\mathrm{TM}}$ FV1000 confocal microscope. HeLa cells expressing SGLP and vector control were labeled with $250 \mathrm{nM}$ Mitotracker at $37^{\circ} \mathrm{C}$ for $15 \mathrm{~min}$. The cells were then fixed, permeabilized and stained with $50 \mu \mathrm{g} / \mathrm{ml}$ fluorescein isothiocyanate (FITC)-phalloidin at room temperature for $2 \mathrm{~h}$ followed by DAPI counterstaining. The ImageJ plugins for colocalization analysis are described and may be downloaded at http://fiji.sc/wiki/index.php/Colocalization_Analysis. The colocalization of FLAG-SGLP with Stat 3 was assessed with the Image J plugin as described by Fay et al (22) and the Pearson's correlation coefficients were also calculated using 
ImageJ. For FRET analysis, images were recorded in three channels for donor, acceptor and transfer, respectively. The donor and acceptor bleed-through was determined and the pseudocolor image of FRET was captured using the ImageJ plugin 'FRET and Colocalization Analyzer', as described by Hachet-Hass et al (23). Photobleaching of DsRed-Racl was achieved by continuous excitation at $561 \mathrm{~nm}$ with full-lamp intensity. Images of GFP-SGLP and DsRed-Rac1 were recorded at intervals of $30 \mathrm{sec}$.

Transwell migration assay. Transwell assays were conducted using Transwell migration chambers with an $8-\mu \mathrm{m}$ pore size (Corning Inc., Acton, MA, USA) according to the manufacturer's instructions. HeLa cells $\left(\sim 1 \times 10^{5}\right)$ suspended in $500 \mu 1$ of serum-free DMEM were seeded in each chamber. The migration chamber was placed into a 24 -well plate with $500 \mu \mathrm{l}$ DMEM containing $10 \% \mathrm{FBS}$ and incubated at $37^{\circ} \mathrm{C}$ with $5 \% \mathrm{CO}_{2}$. Following $8 \mathrm{~h}$, cells on the upper surface were removed with a cotton swab. The migrated cells on the lower surface were fixed and stained with $1 \%$ crystal violet. Five microscopic fields (magnification, x200) were counted per filter in three independent experiments.

BrdU incorporation by flow cytometry. HeLa cells were incubated with $50 \mu \mathrm{M} \mathrm{BrdU}$ at $37^{\circ} \mathrm{C}$ for $30 \mathrm{~min}$ followed by fixation in $80 \%$ ethanol at $4^{\circ} \mathrm{C}$ overnight. The cells were sequentially incubated with $2 \mathrm{M} \mathrm{HCl}$ (room temperature, $30 \mathrm{~min}$ ) and $0.1 \mathrm{M} \mathrm{Na}_{2} \mathrm{~B}_{4} \mathrm{O}_{7}$ buffer ( $\mathrm{pH}$ 8.5) for DNA denaturation. Following extensive washing, the cells were immunostained at room temperature with an anti-BrdU antibody (1:50) for $2 \mathrm{~h}$ and a secondary FITC-conjugated antibody (1:200) for $1 \mathrm{~h}$. The cells were then resuspended in phosphate-buffered saline containing $50 \mu \mathrm{g} / \mathrm{ml}$ propidium iodide and $125 \mu \mathrm{g} / \mathrm{ml}$ RNAase, prior to flow cytometry.

Statistical analysis. Analysis of data from three independent experiments were conducted using GraphPad Prism 5.0 software (GraphPad Software, Inc., La Jolla, CA, USA) and expressed as the mean \pm SD. Student's t-test was used for comparisons among groups. $\mathrm{P}<0.05$ was considered to indicate a statistically significant difference.

\section{Results}

SGLP induces actin filament reorganization in HeLa cells. As SGLP possesses a WxxxE motif, which is required for a number of bacterial virulence factors in subverting host cellular actin cytoskeleton, the actin filament patterns in $\mathrm{HeLa}$ cells expressing SGLP or vector control by FITC-phalloidin staining were examined. The actin cytoskeleton was observed to be markedly altered following transduction of HeLa cells with lentiviral particles expressing SGLP, which was characterized by reduced stress fibers and increased lamellipodia and filopodia (Fig. 1). As the Rho family members of small GTPases are key regulators of stress fibers, lamellipodia and filopodia (24), this result indicated that SGLP is associated with functions of host Rho small GTPases.

SGLP interacts with host Racl and Stat3. The results presented in Fig. 1 revealed that SGLP, which resembles a number of bacterial virulence factors that contain a WxxxE motif, was associated with Rho-family small GTPases. One of the members of Rho small GTPases is Rac1, which is a binding partner of Stat3 (8). In addition, using computational analysis, SGLP was observed to possess a coiled-coil domain that may interact with the corresponding coiled-coil domain of Stat3 (20). Therefore, it was hypothesized that there are probable functional and/or physical interactions of SGLP with Rac1 and Stat3. Thus, a possible interaction between SGLP and Rac1 and Stat3 was investigated.

A GST pull-down assay revealed that the purified GST-SGLP protein binds to host endogenous Rac1 and Stat3 (Fig. 2A). In addition, in HeLa cells, endogenous Rac1 and Stat3 were co-immunoprecipitated with GFP-SGLP using a GFP antibody (Fig. 2B). Immunofluorescence analyses revealed that FLAG-tagged SGLP colocalized with Stat 3 in the nucleus and cytoplasm (Fig. 2C). The Pearson's correlation coefficient of colocalization between SGLP and Stat 3 was $\sim 0.75$ as measured by ImageJ, whereas that of the control was $<0.2$ in 9 cells from three independent experiments. GFP-SGLP also colocalized with DsRed-Rac1 in HeLa cells coexpressing these two proteins (Fig. 2D). Although the DsRed-Racl distribution was homogeneous throughout the cell, which makes it difficult to interpret for colocalization directly, the FRET between GFP-SGLP and DsRed-Rac1 revealed a significant colocalization of SGLP and Rac1 compared with the control (Fig. 2D). Furthermore, consecutive confocal images showed that the GFP-SGLP (donor) intensity increased following photobleaching of the DsRed-Rac1 (acceptor) (Fig. 2E). A linear correlation was observed between the intensity of donor recovery vs. acceptor photobleaching, indicating that GFP-SGLP interacts with DsRed-Rac1 (Fig. 2F).

WxxxE motif is required for SGLP-induced activation of Racl and phosphorylation of Stat3. The results in Fig. 2 revealed that SGLP interacts with Rac1 and Stat3, therefore the study focused on the effect of SGLP on the function of Rac1 and Stat3. As the WxxxE motif is required for a number of bacterial virulence factors to activate cellular Rho-family small GTPases, including Rac1 (16), it was hypothesized that SGLP may also activate Racl and that the WxxxE motif is crucial for this effect. Therefore the study investigated whether SGLP activates Rac1, as well as whether the WxxxE motif is responsible for the activation of Rac1. The wild-type (wt) WVLGE sequence within wt SGLP was mutated to AVLGA to generate an SGLP mutant. HeLa cell lines stably expressing wt SGLP, SGLP mutant or vector, as well as parental HeLa cells were studied. Active Rac1 levels were determined by a GST-Pak1 pull-down assay followed by western blot analysis. In the presence of the WxxxE motif, wt SGLP induced greater Racl activation in HeLa cells as compared with the SGLP mutant and the vector control, as well as the parental cells (Fig. 3A and C). In addition, western blot analysis revealed that wt SGLP increased the phosphorylation of Stat 3 at tyrosine-705 residue (p-Stat3) in HeLa cells and that the SGLP mutant did not exhibit such a prominent effect on Stat3 phosphorylation (Fig. 3B and D).

SGLP-induced Stat3 activation is dependent upon Racl activity. Fig. 3 shows that wt SGLP increased cellular p-Stat3 levels, which reflects its role in the activation of Stat 3 . Thus, 

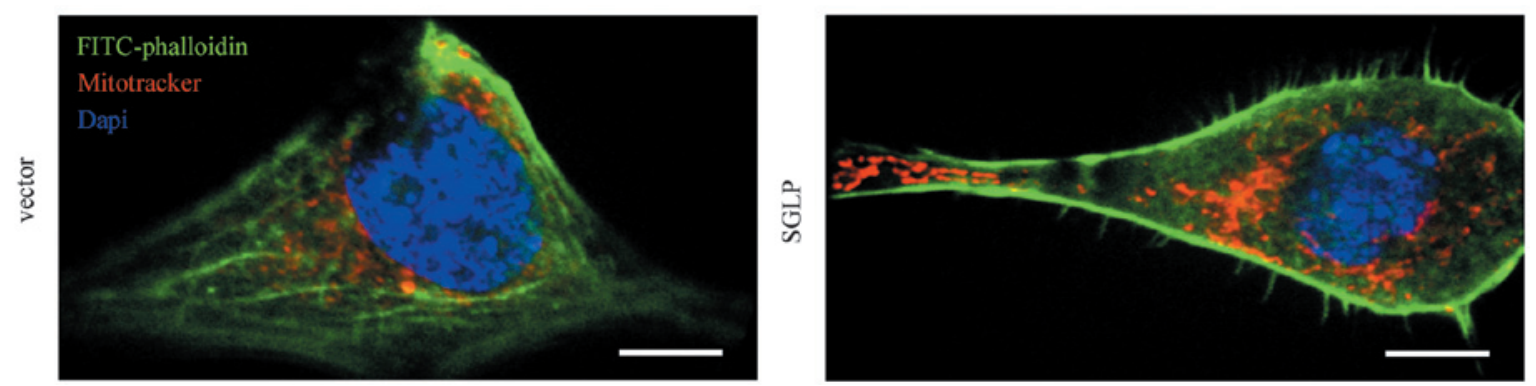

Figure 1. SGLP induces reorganization of actin cytoskeleton in HeLa cells. Confocal images of actin cytoskeleton in HeLa cells expressing SGLP or vector control. The cells were stained by fluorescein isothiocyanate-phalloidin and counterstained with mitotracker and DAPI. Scale bar, $10 \mu \mathrm{m}$. SGLP, small GTPase-like protein fragment.

A

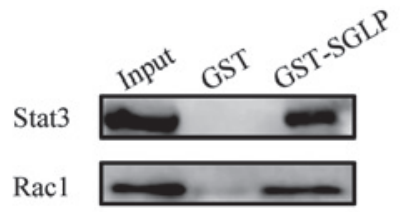

GST-SGLP

GST
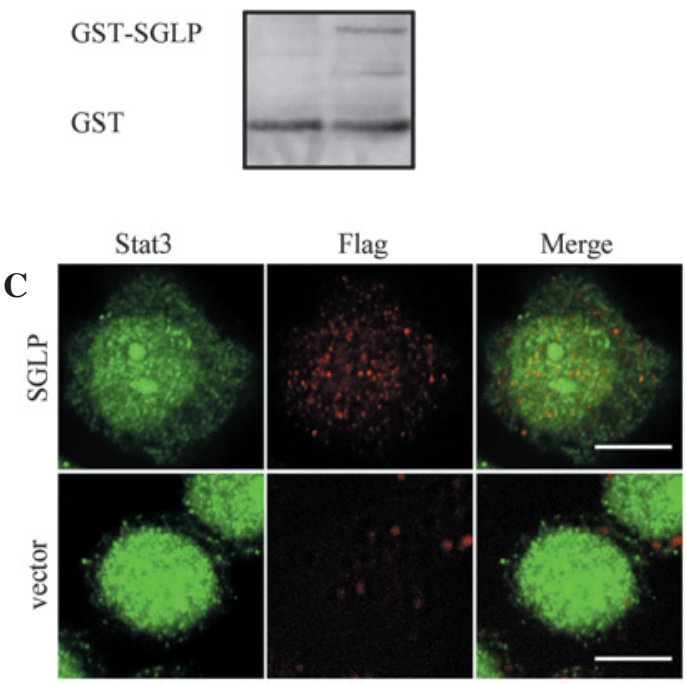

B
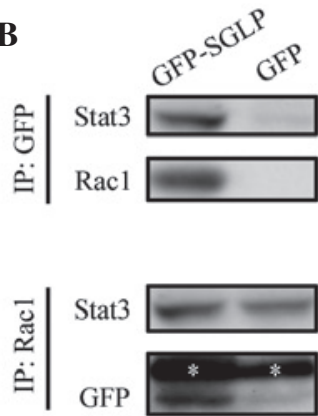

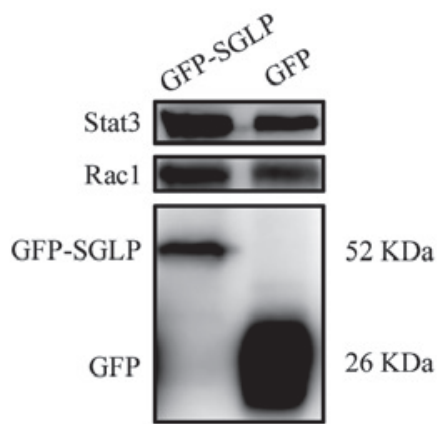

$52 \mathrm{KDa}$

$6 \mathrm{KDa}$
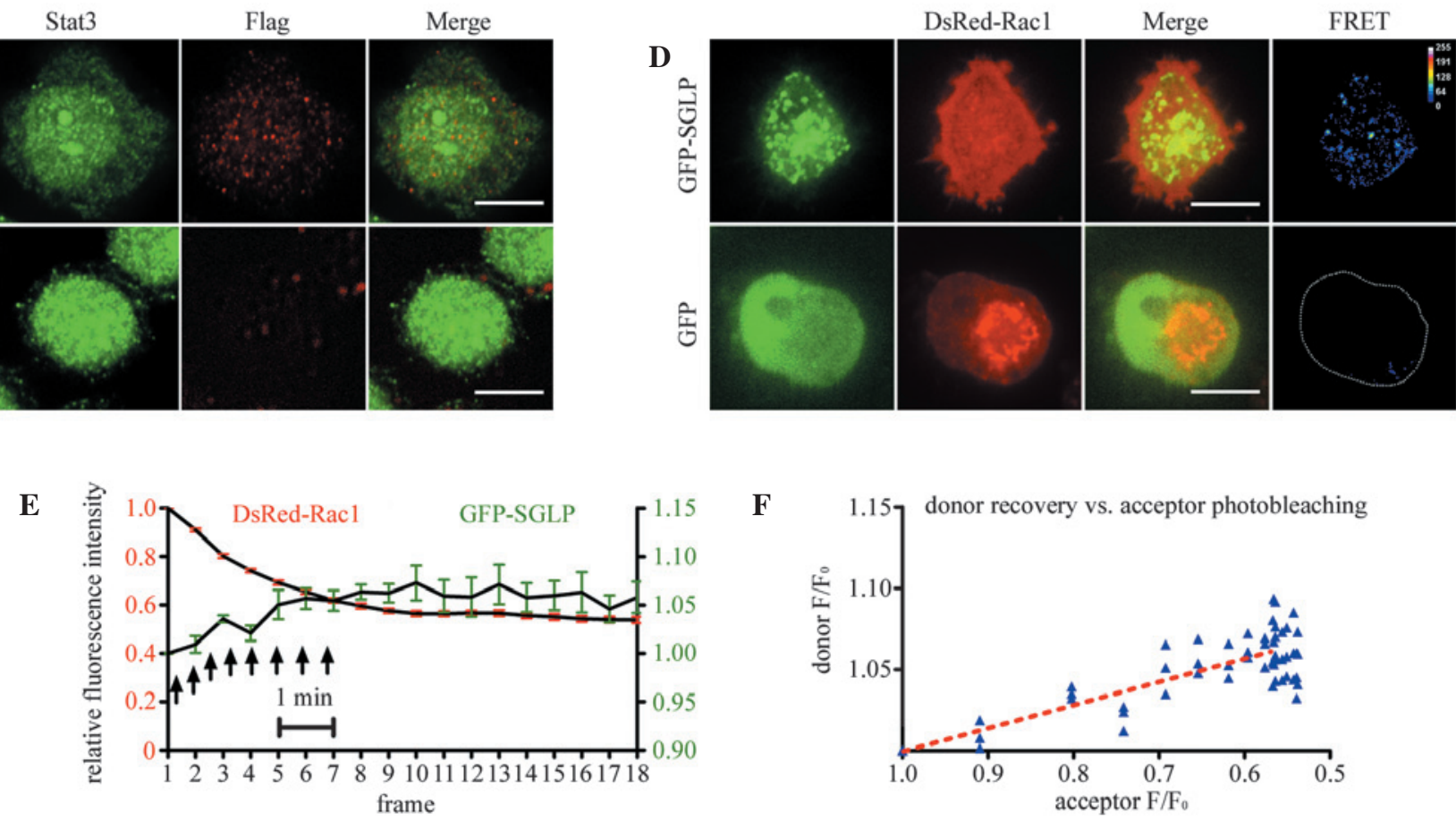

Figure 2. SGLP interacts with Rac1 and Stat3. (A) GST-tagged SGLP ( 10 $\mu \mathrm{g})$ was used to pull-down endogenous Rac1 and Stat3 from IL-6 stimulated HeLa cell lysates $(2 \mathrm{mg})$. The bait protein, GST-SGLP and control GST were also immunoblotted with a GST antibody. (B) HeLa cell lysates expressing GFP-SGLP were immunoprecipitated with a GFP antibody and then probed with anti-Stat3 and anti-Rac1 antibodies. The cell lysates were also immunoprecipitated with a Rac1 antibody and then probed with anti-Stat3 and anti-GFP antibodies. The white asterisk indicates the location of the heavy chain. The right panel shows the cell lysates of input probed with indicated antibodies. (C) HeLa cells expressing FLAG-SGLP were fixed, permeabilized and immunostained with anti-Stat3 (rabbit) and anti-FLAG (mouse) antibodies in conjugation with Alexa Fluor 488 and 594 secondary antibodies, respectively. The confocal images were analyzed for colocalization using an ImageJ plugin. The boxed region is enlarged. Scale bar, $10 \mu \mathrm{m}$. (D) HeLa cells coexpressing GFP-SGLP and DsRed-Rac1 were fixed in $4 \%$ paraformaldehyde and observed by confocal microscopy. As DsRed-Racl was homogenously expressed, the FRET between GFP-SGLP and DsRed-Rac1 was measured, and the FRET indices of GFP-SGLP and GFP control were plotted as pseudocolor images. Scale bar, $10 \mu \mathrm{m}$. (E) HeLa cells, as in (D), were observed by confocal microscopy following photobleaching of DsRed-Rac1. Images were recorded for DsRed-Rac1 (red) and GFP-SGLP (green) every $30 \mathrm{sec}$ during and following photobleaching, and the relative fluorescence intensities were plotted. The black arrows indicate the duration of full-lamp excitation. The negative GFP control did not exhibit such a recovery following photobleaching of DsRed-Rac1 (data not shown). Bar represents \pm SD of nine cells from three independent experiments. $(\mathrm{F})$ A plot of the linear correlation of donor recovery vs. acceptor photobleaching from (E). $\mathrm{F}_{0}$ represents the original fluorescence intensity. SGLP, small GTPase-like protein fragment; GST, glutathione S-transferase; IL, interleukin; GFP, green fluorescent protein. 
A

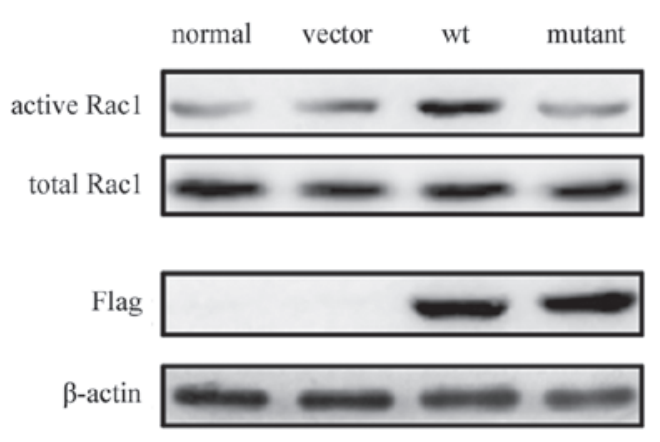

C

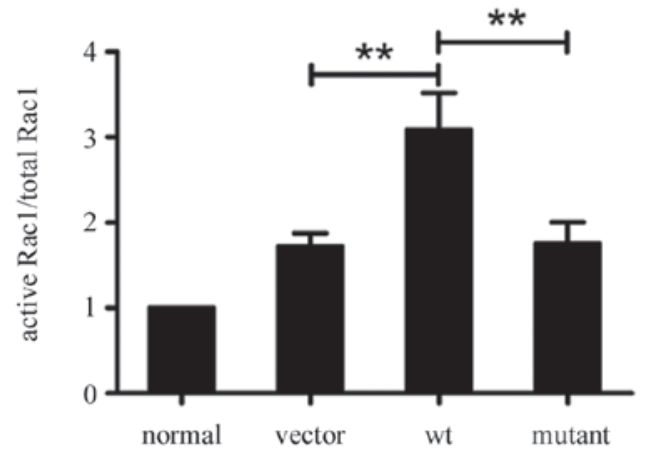

B
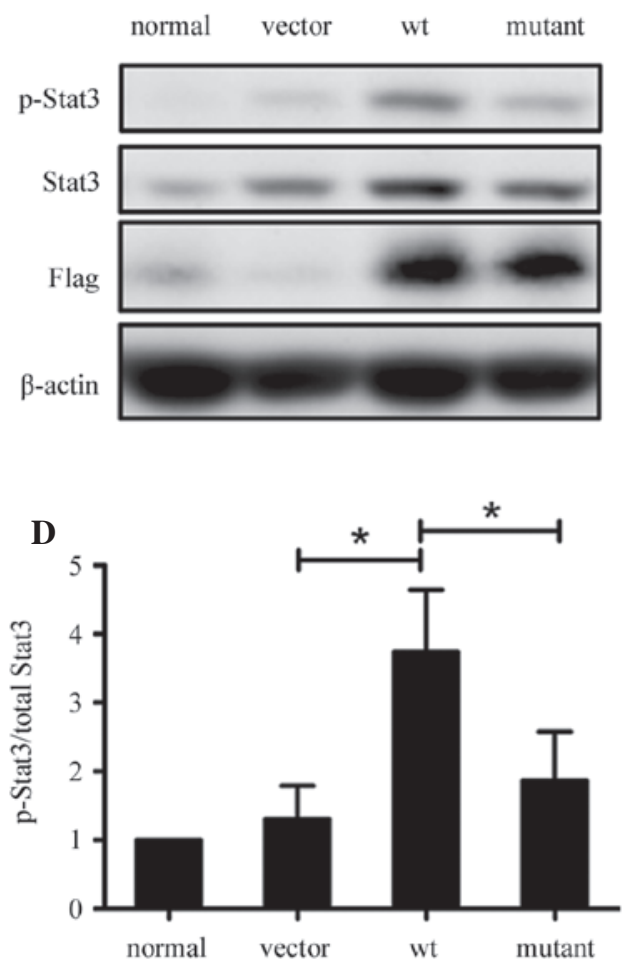

Figure 3. The WxxxE motif of SGLP is required for activation of Rac1 and Stat3. The WVLGE sequence of the wt SGLP was mutated to AVLGA to generate a SGLP mutant. HeLa cells stably expressing FLAG-tagged wt SGLP, SGLP mutant or vector control were studied in addition to the parental cells (normal). (A) A GST-Pak1 pull-down assay was used to determine the relative levels of Rac1 activation. (B) Western blot analysis of phosphorylated Stat3 (tyrosine-705; p-Stat3) and total Stat3. The densitometric ratio of (C) active-Rac1/total-Rac1 and (D) p-Stat3/total-Stat3 from three independent experiments are plotted and normalized to that of the parental cells (normal), which was set to 1 . The error bar represents $\pm \mathrm{SD} ; \mathrm{t}$-test, $\mathrm{n}=3 ;{ }^{*} \mathrm{P}<0.05$ and ${ }^{* *} \mathrm{P}<0.01$, compared with vector and mutant groups, separately. wt, wild-type; SGLP, small GTPase-like protein fragment.

A
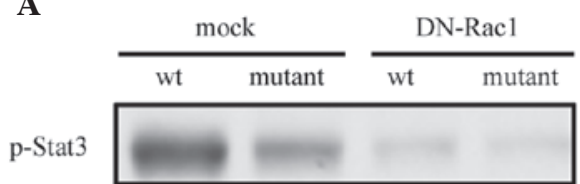

Stat3
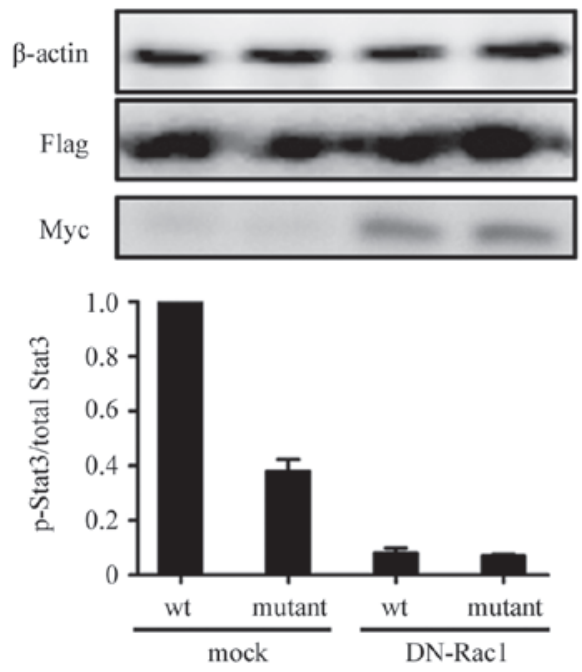

B

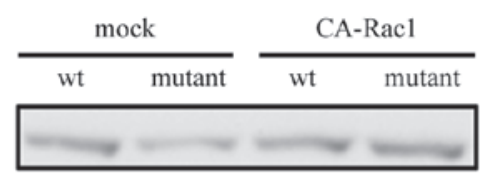

Stat3
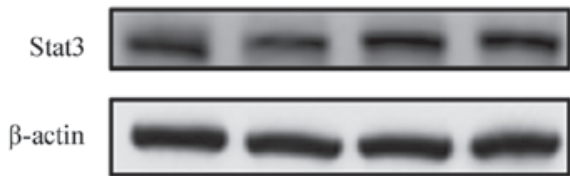

Flag

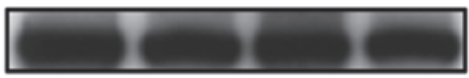

Myc
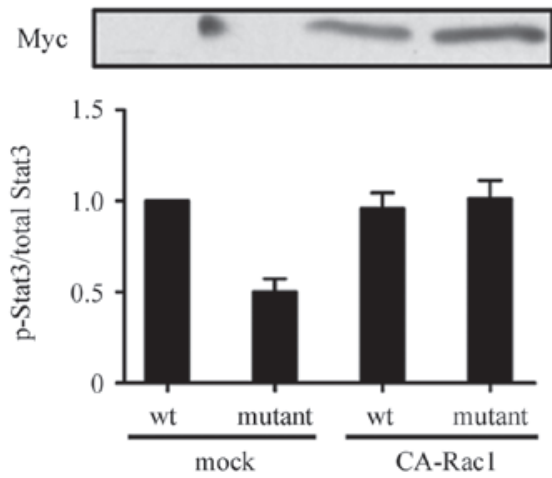

Figure 4. SGLP-induced Stat3 activation is dependent on Rac1 activity. HeLa cells stably expressing FLAG-tagged wt SGLP or SGLP mutant were transfected with either Myc-tagged (A) DN-Racl or (B) CA-Rac1 plasmids for $48 \mathrm{~h}$ and the cells transfected with p-RK5 vector plasmid were used as a control (mock). Cell lysates were probed with the indicated antibodies. In the lower panels, the densitometric ratios of p-Stat $3 /$ total-Stat 3 from three independent experiments were plotted. The ratio of p-Stat3/total-Stat3 in wt SGLP-transduced HeLa cells from mock group was set to 1. The error bar represents \pm SD. SGLP, small GTPase-like protein fragment; DN-Rac1, dominant negative Rac1; CA-RAC1 constitutively active Rac1; wt, wild-type. 


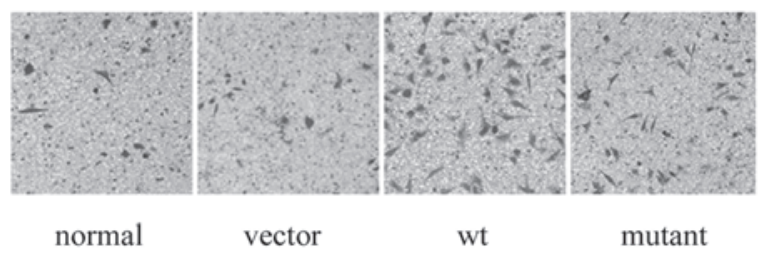

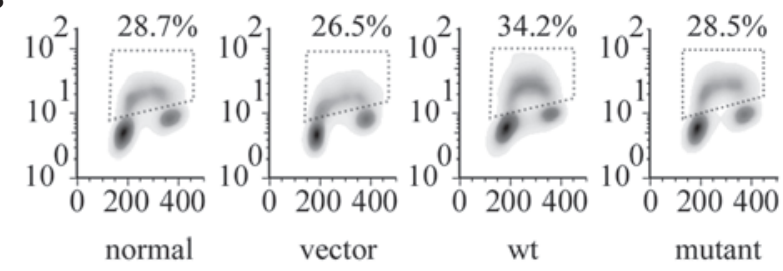
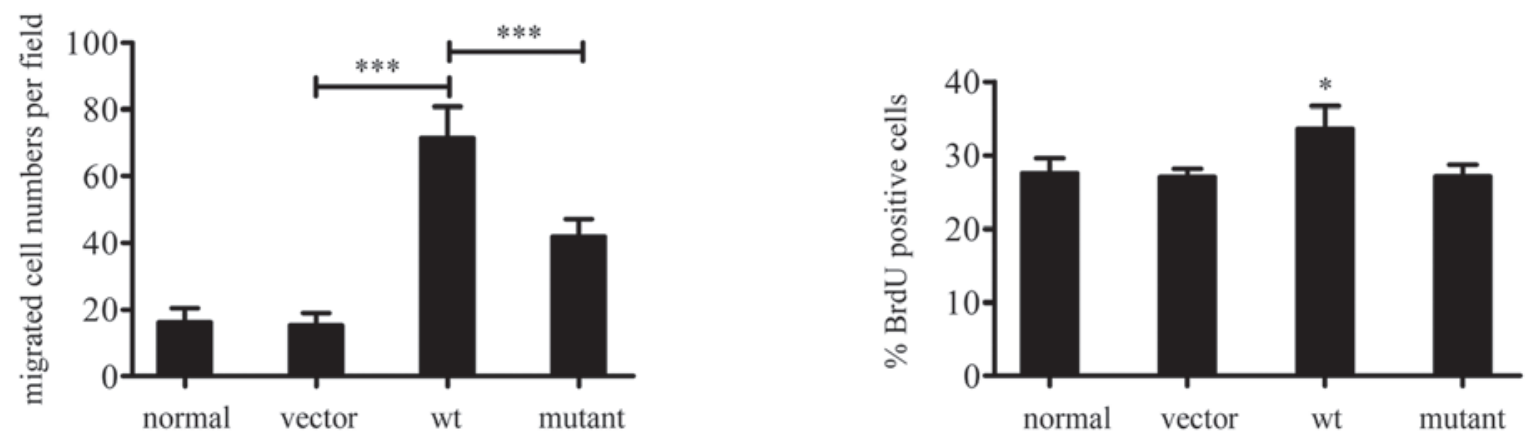

Figure 5. SGLP promotes the migration and proliferation of HeLa cells. HeLa cells stably expressing wt SGLP, SGLP mutant or vector control were observed in addition to the parental cells (normal). (A) Cells were subjected to a Transwell assay to measure cell migration. One representative result is shown (upper panel) and the average of three independent experiments is plotted (lower panel). (B) BrdU incorporation was measured by flow cytometry. The positive cell population was gated and one representative result is shown (upper panel). The average percentage of BrdU-positive cells from three independent experiments is plotted (lower panel). The error bar represents $\pm \mathrm{SD}$; t-test, $\mathrm{n}=3 ;{ }^{*} \mathrm{P}<0.05$, compared with other three groups, separately; ${ }^{* * * *} \mathrm{P}<0.001$, compared with vector and mutant groups, separately.. wt, wild-type; SGLP, small GTPase-like protein fragment.

the mechanism by which SGLP activates Stat3 was investigated. As Racl mediates Stat3 activity through direct binding to Stat3 and an indirect activation loop of autocrine IL-6 $(8,25)$, it was examined whether the SGLP-induced activation of Stat3 was dependent on Rac1 activity. The wt and mutant SGLP-transduced HeLa cells were transfected with dominant negative Rac1 (DN-Rac1) or constitutive active Rac1 (CA-Rac1) plasmids separately. Overexpression of DN-Racl was observed to eliminate the increase in p-Stat3 levels in wt SGLP-transduced HeLa cells (Fig. 4A), whereas overexpression of CA-Racl rescued the decrease in p-Stat3 levels in mutant SGLP-transduced HeLa cells (Fig. 4B). The results suggested that SGLP activates Stat3 depending on the active form of Rac1.

SGLP promotes migration and proliferation of HeLa cells. The aforementioned observations suggest that SGLP activates Rac1 and Stat3. Since Rac1 and Stat 3 are involved in cell migration and proliferation (14), the possibility of whether SGLP may affect migration and/or proliferation of HeLa cells was determined. In a Transwell assay, wt SGLP was observed to increase the transwell migration of HeLa cells relative to the control and SGLP (mutant) (Fig. 5A), which is consistent with the levels of active Racl demonstrated in Fig. 3A. These observations suggest that the effect of wt SGLP on HeLa cell migration is associated with the wt SGLP-induced Rac1 activation. BrdU incorporation rates in HeLa cells were then investigated by flow cytometry. Wt SGLP was observed to promote BrdU incorporation (Fig. 5B), which suggests that SGLP may exert a pro-proliferative effect in HeLa cells.

\section{Discussion}

In the current study, SGLP, an N terminal protein fragment of Smc from Mycoplasma pulmonis, was identified as a mycoplasmal virulence factor. Homologues of SGLP may be observed in a minimum of 25 Mycoplasma species, which exhibit high levels of homology around the sequences encompassing the WVLGE motif. This conservation in evolution reflects that SGLP and its homologues may be considered as a group of common virulence factors of Mycoplasma species.

The study observed that SGLP-transduced HeLa cells exhibited reduced stress fibers and increased filopodia and lamellipodia. These phenotypic changes in actin filament distribution may have resulted from alterations in Rho-family small GTPase activity $(26,27)$. The host Rho-family small GTPases are hypothesized to be involved in SGLP-induced cellular alterations. An interaction between SGLP and members of Rho-family small GTPases, including Rac1 was hypothesized. In addition, Stat 3 directly binds to Rac1 and modulates its function $(8,13)$. The possibility of SGLP interacting with Rac1 and/or Stat3 was investigated. SGLP was observed to form a protein complex with Rac1 and Stat3, which suggests that SGLP may interact directly with Rac1 and Stat3. SGLP was also confirmed to colocalize with Rac1 and Stat 3 in the nucleus and cytoplasm, which reflects that SGLP may interact with Rac1 and Stat 3 at various locales within host cells.

As SGLP is a binding partner of Rac1, the effect of SGLP on Rac1 activity was determined. SGLP was observed to increase the active Racl level. Since the WxxxE motif is crucial for a number of bacterial proteins to alter host Rho-family small GTPase activity (16-18), SGLP was hypothesized to cause a similar effect on Rac1 depending on the WxxxE motif. The SGLP mutant with a mutated AxxxA sequence did not induce Rac1 activation, which is consistent with the hypothesis. As active Racl promotes cell migration (28), it was assumed that SGLP-induced Rac1 activation may also contribute to tumor cell migration. SGLP was observed to increase cell migration 
of tumor cells in vitro, while the SGLP mutant did not exert this effect. These results confirmed the hypothesis that the WxxxE motif is required for SGLP to increase Racl activity and that SGLP-induced Rac1 activation may be responsible for the observed increase in cell migration. However, this explanation does not exclude other small GTPases that may also contribute to SGLP-associated increase in tumor cell migration.

Stat 3 activation is involved in a number of signaling pathways and it is pivotal in receptor tyrosine kinase-mediated pro-proliferative signal transduction (29). SGLP was observed to induce Stat 3 activation. SGLP was also identified to exhibit a pro-proliferative effect on tumor cells in terms of BrdU incorporation rates, which is consistent with the observed SGLP-induced activation of Stat3. In view of the dependency of SGLP-induced Stat 3 activation on Rac1 activity, it was hypothesized that SGLP may induce Rac1 activation, which, in turn, contributes to Stat 3 activation. Although the role of exact domains of SGLP in forming the protein complex with Rac1 and Stat 3 is somewhat unclear, the objective of the current study was to reveal and highlight a molecular mechanism by which Mycoplasma may affect host cellular responses.

In conclusion, the current findings suggest that, through interaction with Rac1 and Stat3, SGLP may potentiate tumor cell migration and proliferation in vitro. However, its involvement in promoting tumor development in vivo remains uncertain. The identification of an individual mycoplasmal virulence factor that interacts with and activates Racl and subsequently triggers Stat 3 activation is novel. Further studies of the role of SGLP in Mycoplasma-associated pathogenesis are likely to contribute to knowledge of Mycoplasma-induced malignant transformation of mammalian cells.

\section{References}

1. Sinkovics JG: Molecular biology of oncogenic inflammatory processes. I. Non-oncogenic and oncogenic pathogens, intrinsic inflammatory reactions without pathogens, and microRNA/DNA interactions (Review). Int J Oncol 40: 305-349, 2012.

2. Zhang S, Tsai S and Lo SC: Alteration of gene expression profiles during mycoplasma-induced malignant cell transformation. BMC Cancer 6: 116, 2006.

3. Tsai S, Wear DJ, Shih JW and Lo SC: Mycoplasmas and oncogenesis: persistent infection and multistage malignant transformation. Proc Natl Acad Sci USA 92: 10197-10201, 1995.

4. Rottem S: Interaction of mycoplasmas with host cells. Physiol Rev 83: 417-432, 2003.

5. Choi SY, Lim JW, Shimizu T, Kuwano K, Kim JM and Kim H: Reactive oxygen species mediate Jak2/Stat3 activation and IL-8 expression in pulmonary epithelial cells stimulated with lipid-associated membrane proteins from Mycoplasma pneumoniae. Inflamm Res 61: 493-501, 2012.

6. Rawadi G, Zugaza JL, Lemercier B, et al: Involvement of small GTPases in Mycoplasma fermentans membrane lipoproteins-mediated activation of macrophages. J Biol Chem 274: 30794-30798, 1999.

7. Takai Y, Sasaki T and Matozaki T: Small GTP-binding proteins. Physiol Rev 81: 153-208, 2001

8. Simon AR, Vikis HG, Stewart S, Fanburg BL, Cochran BH and Guan KL: Regulation of STAT3 by direct binding to the Rac1 GTPase. Science 290: 144-147, 2000.

9. Gupta SC, Hevia D, Patchva S, Park B, Koh W and Aggarwal BB Upsides and downsides of reactive oxygen species for cancer: the roles of reactive oxygen species in tumorigenesis, prevention, and therapy. Antioxid Redox Signal 16: 1295-1322, 2012.
10. Millonig G, Ganzleben I, Peccerella T, et al: Sustained submicromolar $\mathrm{H}_{2} \mathrm{O}_{2}$ levels induce hepcidin via signal transducer and activator of transcription 3 (STAT3). J Biol Chem 287: 37472-37482, 2012.

11. Tonozuka Y, Minoshima Y, Bao YC, et al: A GTPase-activating protein binds STAT3 and is required for IL-6-induced STAT3 activation and for differentiation of a leukemic cell line. Blood 104: 3550-3557, 2004.

12. Kawashima T, Bao YC, Nomura $\mathrm{Y}$, et al: Rac1 and a GTPase-activating protein, MgcRacGAP, are required for nuclear translocation of STAT transcription factors. J Cell Biol 175: 937-946, 2006.

13. Teng TS, Lin B, Manser E, Ng DC and Cao X: Stat3 promotes directional cell migration by regulating Rac1 activity via its activator betaPIX. J Cell Sci 122: 4150-4159, 2009.

14. Debidda M, Wang L, Zang H, Poli V and Zheng Y: A role of STAT3 in Rho GTPase-regulated cell migration and proliferation. J Biol Chem 280: 17275-17285, 2005.

15. Azare J, Leslie K, Al-Ahmadie H, et al: Constitutively activated Stat3 induces tumorigenesis and enhances cell motility of prostate epithelial cells through integrin beta 6. Mol Cell Biol 27: 4444-4453, 2007.

16. Alto NM, Shao F, Lazar CS, et al: Identification of a bacterial type III effector family with $\mathrm{G}$ protein mimicry functions. Cell 124: 133-145, 2006

17. Bulgin R, Raymond B, Garnett JA, et al: Bacterial guanine nucleotide exchange factors SopE-like and WxxxE effectors. Infect Immun 78: 1417-1425, 2010.

18. Jackson LK, Nawabi P, Hentea C, Roark EA and Haldar K: The Salmonella virulence protein SifA is a $\mathrm{G}$ protein antagonist. Proc Natl Acad Sci USA 105: 14141-14146, 2008.

19. Ham JH, Majerczak DR, Nomura K, et al: Multiple activities of the plant pathogen type III effector proteins WtsE and AvrE require WxxxE motifs. Mol Plant Microbe Interact 22: 703-712, 2009.

20. Hagemann UB, Mason JM, Müller KM and Arndt KM: Selectional and mutational scope of peptides sequestering the Jun-Fos coiled-coil domain. J Mol Biol 381: 73-88, 2008.

21. Luo X, Li Z, Li X, et al: hSav1 interacts with HAX1 and attenuates its anti-apoptotic effects in MCF-7 breast cancer cells. Int J Mol Med 28: 349-355, 2011.

22. Fay FS, Taneja KL, Shenoy S, Lifshitz L and Singer RH: Quantitative digital analysis of diffuse and concentrated nuclear distributions of nascent transcripts, SC35 and poly(A). Exp Cell Res 231: 27-37, 1997.

23. Hachet-Haas M, Converset N, Marchal O, et al: FRET and colocalization analyzer - a method to validate measurements of sensitized emission FRET acquired by confocal microscopy and available as an ImageJ Plug-in. Microsc Res Tech 69: 941-956, 2006.

24. Nobes CD and Hall A: Rho, rac, and cdc42 GTPases regulate the assembly of multimolecular focal complexes associated with actin stress fibers, lamellipodia, and filopodia. Cell 81: 53-62, 1995.

25. Faruqi TR, Gomez D, Bustelo XR, Bar-Sagi D and Reich NC: Rac1 mediates STAT3 activation by autocrine IL-6. Proc Nat Acad Sci USA 98: 9014-9019, 2001.

26. Albertinazzi C, Cattelino A and de Curtis I: Rac GTPases localize at sites of actin reorganization during dynamic remodeling of the cytoskeleton of normal embryonic fibroblasts. J Cell Sci 112: 3821-3831, 1999.

27. Hoang MV, Nagy JA and Senger DR: Active Rac1 improves pathologic VEGF neovessel architecture and reduces vascular leak: mechanistic similarities with angiopoietin-1. Blood 117: 1751-1760, 2011.

28. Migeotte I, Omelchenko T, Hall A and Anderson KV: Rac1-dependent collective cell migration is required for specification of the anterior-posterior body axis of the mouse. PLoS Biol 8: e1000442, 2010.

29. Sehgal PB: Paradigm shifts in the cell biology of STAT signaling. Semin Cell Dev Biol 19: 329-340, 2008. 